\title{
MEANDERING POINTS OF TWO-DIMENSIONAL BROWNIAN MOTION
}

\author{
By MichIo SHIMURA
}

\section{$\S 1$. Introduction and the result.}

Let $Z_{w}(t)=\left(X_{w}(t), Y_{w}(t)\right), w \in W,-\infty<t<\infty$, be the two-dimensional standard Brownian motion with $Z_{w}(0)=0$ on a probability space $(W, \beta, P)$. Let $\vartheta$ denote the set of all unit vectors in $\boldsymbol{R}^{2}$. For every $\boldsymbol{u}$ in $U$ we set a half-plane $H(\boldsymbol{u})=\left\{\boldsymbol{x} \in \boldsymbol{R}^{2} \mid \boldsymbol{u} \cdot \boldsymbol{x} \geqq 0\right\}$, where $\boldsymbol{u} \cdot \boldsymbol{x}$ denotes the inner product. For $\boldsymbol{u}_{1}$ and $\boldsymbol{u}_{2}$ in $U$ we consider the random set of all two-sided meandering times of the Brownian motion:

$$
\begin{aligned}
& \mathscr{M}_{w}\left(\boldsymbol{u}_{1}, \boldsymbol{u}_{2}\right)=\left\{-\infty<t<\infty \mid{ }^{\exists} h>0 \text { such that } Z_{w}(s) \in H\left(\boldsymbol{u}_{1}\right)+Z_{w}(t) \text { for } t-h<{ }^{{ }} s<t\right. \\
& \left.\& \quad Z_{w}(s) \in H\left(\boldsymbol{u}_{2}\right)+Z_{w}(t) \text { for } t<{ }^{\forall} s<t+h\right\} .
\end{aligned}
$$

In this paper we will prove the following theorem.

THEOREM 1. For every $\boldsymbol{u}_{1}$ and $\boldsymbol{u}_{2}$ in $\vartheta$ with $\boldsymbol{u}_{1} \neq \boldsymbol{u}_{2}$, we have $\mathfrak{M}_{w}\left(\boldsymbol{u}_{1}, \boldsymbol{u}_{2}\right)=\varnothing$ almost surely (a.s. for abbreviation).

Our problem arises from the following observation. By a result of Evans [2] we have $\operatorname{dim} \mathscr{M}_{w}\left(\boldsymbol{u}_{1}, \boldsymbol{u}_{2}\right)=1-\pi / 2 \pi-\pi / 2 \pi=0$ a. s.. Here we note that, in such a critical case, we do not know from the result whether the set is empty or not (a.s.). Indeed, both cases may occur: Obviously $\mathscr{M}_{w}(\boldsymbol{u}, \boldsymbol{u}) \neq \varnothing$ a. s. ; $\mathscr{M}_{w}(\boldsymbol{u},-\boldsymbol{u})=\varnothing$ a.s. from Dvoretzky, Erdös and Kakutani [1] on the nonexistence of points of increase (decrease) for the one-dimensional Brownian motion. So, it may be interesting to see if the set is empty or not (a.s.) for $\boldsymbol{u}_{1} \neq \boldsymbol{u}_{2}$. By Theorem 1 we answer the problem. As will be shown in the following sections, the proof of Theorem 1 in [1] still works to ours by some modification.

The paper is organized as follows. In $\S 2$ we give preliminaries to our proof of Theorem 1 . We show in $\S 3$ two lemmas which play key role in $\S 4$, where the proof of Theorem 1 is given.

Received September 16, 1987 


\section{$\S 2$. Preliminaries.}

We may take in Theorem $1 \quad \boldsymbol{u}_{1}=(0,1)$ and $\boldsymbol{u}_{2}=(-\sin \theta, \cos \theta), 0<\theta \leqq \pi$, without loss of generality. We write $\mathscr{M}_{w}=\mathscr{M}_{w}\left(\boldsymbol{u}_{1}, \boldsymbol{u}_{2}\right)$. Besides the $x y$ coordinate system we set the $x^{\prime} y^{\prime}$ one in which the $y^{\prime}$-axis is directed toward the vector $\boldsymbol{u}_{2}$. We put $Z_{w}(t)=\left(X_{w}^{\prime}(t), Y_{w}^{\prime}(t)\right)$ in this system. Note that each of the processes $X_{w}(\cdot), Y_{w}(\cdot), X_{w}^{\prime}(\cdot)$ and $Y_{w}^{\prime}(\cdot)$ is the one-dimensional standard Brownian motion. For $-\infty<s<t<\infty$ we define

$$
\underline{Y}_{w}[s, t]=\min \left\{Y_{w}(u) \mid s \leqq u \leqq t\right\} \quad \text { and } \quad \bar{Y}_{w}[s, t]=\max \left\{Y_{w}(u) \mid s \leqq u \leqq t\right\}
$$

$\left(\underline{Y}_{w}^{\prime}[s, t], \bar{Y}_{w}^{\prime}[s, t]\right.$, etc are defined in the same way).

We put

$$
A=\left\{\left.w \in W\right|^{\exists} t \in[0,1] \text { such that } \underline{Y}_{w}[t-2, t] \geqq Y_{w}(t) \quad \& \quad Y_{w}^{\prime}(t) \leqq \underline{Y}_{w}^{\prime}[t, t+2]\right\} .
$$

It is easy to see that Theorem 1 follows if we have $P(A)=0$. For $n \geqq 1$ and $1 \leqq k \leqq 2 n$ we set

$$
\begin{aligned}
& A_{k}^{n}=\{w \in W \mid \underline{Y}_{w}\left[\frac{k}{n}-2, \frac{k-1}{n}\right] \geqq \underline{Y}_{w}\left[\frac{k-1}{n}, \frac{k}{n}\right] \\
&\left.\& \quad \underline{Y}_{w}^{\prime}\left[\frac{k-1}{n}, \frac{k}{n}\right] \leqq \underline{Y}_{w}^{\prime}\left[\frac{k}{n}, \frac{k-1}{n}+2\right]\right\} .
\end{aligned}
$$

Then $A=\bigcap_{n=1}^{\infty} \bigcup_{k=1}^{n} A_{k}^{n}$. Here we note a modification made in the definition of the sets $A$ and $A_{k}^{n}$ from those given in (5.5) and (5.6) in [1]. Such a change will be necessary to treat the case $\boldsymbol{u}_{1} \neq \boldsymbol{u}_{2}$. Put

$$
S_{k}^{n}=S_{k}^{n}(w)=\sum_{j=1}^{k} 1_{w}\left(A_{\jmath}^{n}\right),
$$

where $1_{w}(A)$ is the indicator function on a set $A(\subseteq W)$. Since $P(A) \leqq P\left(S_{n}^{n} \geqq 1\right)$ for all $n$, we will prove as in [1]

$$
P\left(S_{n}^{n} \geqq 1\right) \leqq \frac{E\left(S_{2 n}^{n}\right)}{E\left(S_{2 n}^{n} \mid S_{n}^{n} \geqq 1\right)} \rightarrow 0 \quad \text { as } n \rightarrow \infty
$$

in $\S 4$ to get Theorem 1 .

\section{§ 3. Two lemmas.}

Before showing the lemmas we list some formulas which will be used often later. Put

$$
\Phi_{t}(\xi)=\left(\frac{2}{\pi t}\right)^{1 / 2} \int_{0}^{\xi} \exp \left(-\frac{x^{2}}{2 t}\right) d x \text { for } t>0 \text { and } \xi \geqq 0 .
$$

It is well-known that, for $t>0$ and $\xi \leqq 0$,

$$
P\left(\underline{Y}_{w}[-t, 0] \geqq \xi\right)=P\left(\underline{Y}_{w}[0, t] \geqq \xi\right)=\Phi_{t}(-\xi)
$$


((3.1) also holds by replacing the process $Y$ by another one, e.g., the $\left.Y^{\prime}\right)$. It holds from the inequalities $1-x \leqq \exp (-x) \leqq 1$ for $x \geqq 0$ the following:

$$
\Phi_{t}(\xi) \leqq\left(\frac{2}{\pi t}\right)^{1 / 2} \int_{0}^{\xi} d x=\left(\frac{2}{\pi t}\right)^{1 / 2} \xi \text { for } \xi \geqq 0
$$

and

$$
\Phi_{t}(\xi) \geqq\left(\frac{2}{\pi t}\right)^{1 / 2} \int_{0}^{\xi}\left(1-\frac{x^{2}}{2 t}\right) d x \geqq(2 \pi t)^{-1 / 2} \xi \text { for } 0 \leqq \xi \leqq(3 t)^{1 / 2}
$$

Moreover

$$
1-\Phi_{t}(\xi)<\left(\frac{2}{\pi t}\right)^{1 / 2} \int_{\xi}^{\infty} x \exp \left(-\frac{x^{2}}{2 t}\right) d x=\left(\frac{2}{\pi t}\right)^{1 / 2} \exp \left(-\frac{\xi^{2}}{2 t}\right) \text { for } \xi \geqq 1
$$

Let $f(s) \asymp s^{-\alpha}(s \rightarrow c)$ denote

$$
0<\liminf _{s \rightarrow c} s^{\alpha} f(s) \leqq \limsup _{s \rightarrow c} s^{\alpha} f(s)<\infty .
$$

Firstly we show the following lemma.

LEMMA 1. We have $P\left(A_{1}^{n}\right) \asymp n^{-1}(n \rightarrow \infty)$.

Proof. Set $H^{n}(d \xi, d \eta)=P\left(\underline{Y}_{w}[0,1 / n] \in d \xi \quad \& \quad \underline{Y}_{w}^{\prime}[0,1 / n]-Y_{w}^{\prime}(1 / n) \in d \eta\right)$. Since the Brownian motion has independent and stationary increments, the following identity holds :

$$
\begin{aligned}
P\left(A_{1}^{n}\right)= & P\left(\underline{Y}_{w}\left[\frac{1}{n}-2,0\right] \geqq \underline{Y}_{w}\left[0, \frac{1}{n}\right] \&\right. \\
& \left.\underline{Y}_{w}^{\prime}\left[0, \frac{1}{n}\right]-\underline{Y}_{w}^{\prime}\left(\frac{1}{n}\right) \leqq \underline{Y}_{w}^{\prime}\left[\frac{1}{n}, 2\right]-Y_{w}^{\prime}\left(\frac{1}{n}\right)\right) \\
= & \int_{-\infty}^{0} \int_{-\infty}^{0} P\left(\underline{Y}_{w}\left[\frac{1}{n}-2,0\right] \geqq \xi\right) P\left(\underline{Y}_{w}^{\prime}\left[0,2-\frac{1}{n}\right] \geqq \eta\right) H^{n}(d \xi, d \eta) .
\end{aligned}
$$

Therefore, together with the scaling relation $H^{n}(d \xi, d \eta)=H^{1}\left(n^{1 / 2} d \xi, n^{1 / 2} d \eta\right)$, we conclude from (3.1), (3.2) and from (3.3) the following:

$$
P\left(A_{1}^{n}\right) \leqq \int_{-\infty}^{0} \int_{-\infty}^{0} \Phi_{1}(-\xi) \Phi_{1}(-\eta) H^{n}(d \xi, d \eta) \leqq \frac{2}{\pi n} \int_{-\infty}^{0} \int_{-\infty}^{0}|\xi \eta| H^{1}(d \xi, d \eta)
$$

and

$$
\begin{aligned}
P\left(A_{1}^{n}\right) & \geqq \int_{-6^{1 / 2}}^{0} \int_{-6^{1 / 2}}^{0} \Phi_{2}(-\xi) \Phi_{2}(-\eta) H^{n}(d \xi, d \eta) \\
& \geqq \frac{1}{4 \pi n} \int_{-(6 n)^{1 / 2}}^{0} \int_{-(6 n)^{1 / 2}}^{0}|\xi \eta| H^{1}(d \xi, d \eta)
\end{aligned}
$$

(note $\left.0<\int_{-\infty}^{0} \int_{-\infty}^{0}|\xi \eta| H^{1}(d \xi, d \eta)<\infty\right)$. This proves the lemma. 
Let $G(\Delta, \delta), 0 \leqq \Delta, \delta$, denote the probability

$$
P\left(\underline{Y}_{w}^{\prime}[0,1] \geqq-\Delta \& Y_{w}^{\prime}(1) \geqq 1 \& \underline{Y}_{w}[0,1] \geqq Y_{w}(1)-\delta \& Y_{w}(1) \leqq-1\right) .
$$

Next we show the following lemma.

LEMma 2. There exists a positive constant $K$ such that the following holds:

$$
G(\Delta, \delta) \geqq K \Delta \delta \text { for every } 0 \leqq \Delta, \delta \leqq 1 .
$$

Proof. Let us consider the case $0<\theta \leqq \pi / 2$. Take the point $A$ where the lines $y=-2$ and $y^{\prime}=-1$ cross each other. Note that the $x^{\prime}$ co-ordinate of $A$, say $a$, is less than -1.73 . Let $B$ denote the point of intersection of the lines $y=-4$ and $y^{\prime}=1$, and put by $b$ its $x$ co-ordinate. Take a disc $D$ of diameter $1 / 2$ contained in the region $\{z=(x, y) \mid x<b\} \cap\left\{z=\left(x^{\prime}, y^{\prime}\right) \mid a<x^{\prime}<0\right\}$. Then, an elementary geometric consideration, together with the independence and the stationarity of increments of the Brownian motion, lead us to the following estimate (see Figure 1 below):

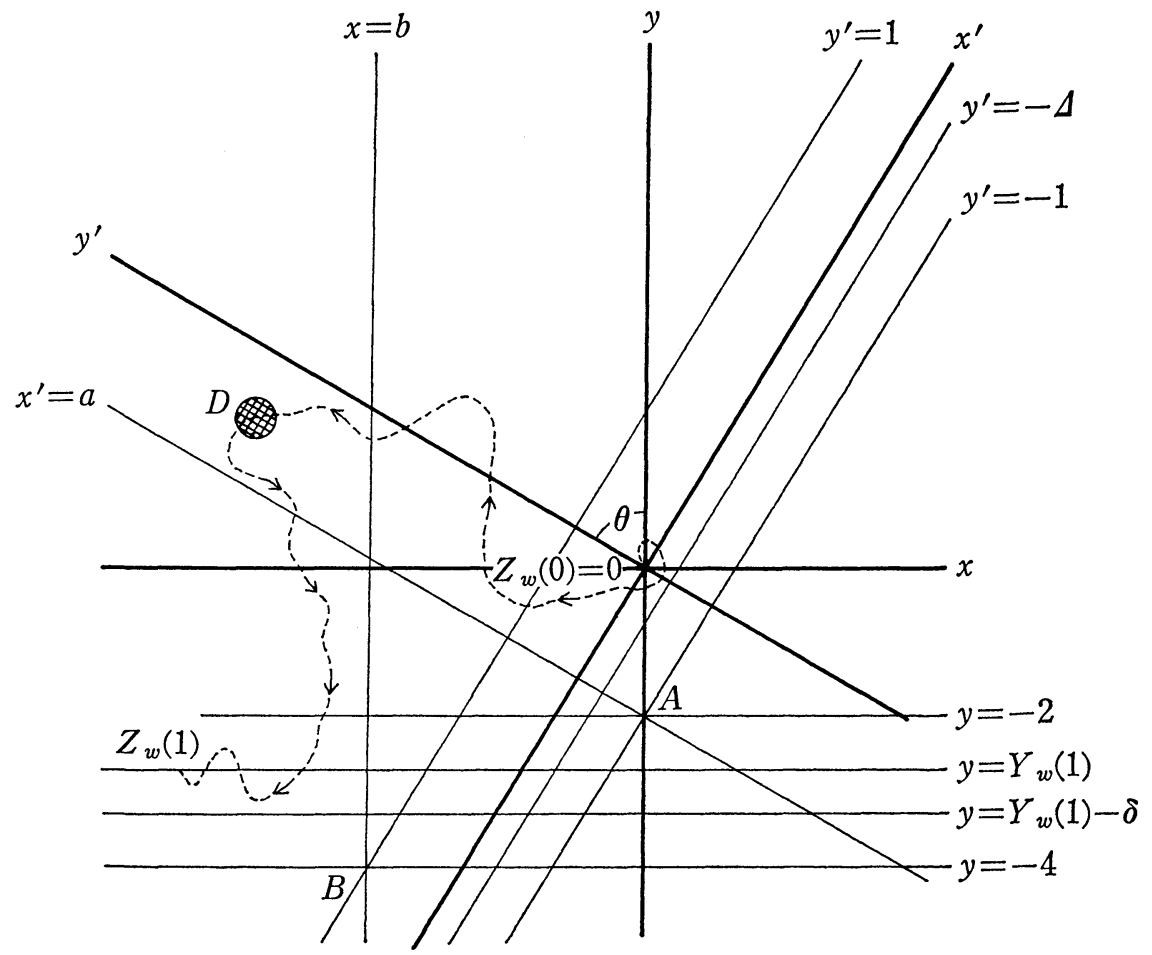

Figure 1 


$$
\begin{aligned}
& G(\Delta, \delta)>P\left(\underline{Y}_{w}^{\prime}\left[0, \frac{1}{2}\right] \geqq-\Delta \& \underline{X}_{w}^{\prime}\left[0, \frac{1}{2}\right] \geqq a \&\right. \\
& Z_{w}\left(\frac{1}{2}\right) \in D \& \underline{Y}_{w}\left[\frac{1}{2}, 1\right] \geqq Y_{w}(1)-\delta \&-3 \leqq Y_{w}(1) \leqq-2 \& \\
& \left.\bar{X}_{w}\left[\frac{1}{2}, 1\right] \leqq b\right)=\iint_{D} P\left(\underline{Y}_{w}^{\prime}\left[0, \frac{1}{2}\right] \geqq-\Delta \& \underline{X}_{w}^{\prime}\left[0, \frac{1}{2}\right] \geqq a \&\right. \\
& \left.\left(X_{w}\left(\frac{1}{2}\right), Y_{w}\left(\frac{1}{2}\right)\right) \in d x \times d y\right) P\left(\underline{Y}_{w}\left[0, \frac{1}{2}\right] \geqq Y_{w}\left(\frac{1}{2}\right)-\delta \&\right. \\
& \left.-3-y \leqq Y_{w}\left(\frac{1}{2}\right) \leqq-2-y \& \bar{X}_{w}\left[0, \frac{1}{2}\right] \leqq b-x\right) .
\end{aligned}
$$

Put $\min \{b-x \mid(x, y) \in D\}=b^{\prime}(>0)$ and $\min \{-2-y \mid(x, y) \in D\}=c$, Then, noting $\max \{-3-y \mid(x, y) \in D\}=c-1 / 2$, we have

$$
\begin{aligned}
& G(\Delta, \delta)>P\left(\underline{Y}_{w}^{\prime}\left[0, \frac{1}{2}\right] \geqq-\Delta \& \underline{X}_{w}^{\prime}\left[0, \frac{1}{2}\right] \geqq a\right. \text { \& } \\
& \left.Z_{w}\left(\frac{1}{2}\right) \in D\right) P\left(\underline{Y}_{w}\left[0, \frac{1}{2}\right] \geqq Y_{w}\left(\frac{1}{2}\right)-\delta \& \quad c-\frac{1}{2} \leqq Y_{w}\left(\frac{1}{2}\right) \leqq c \quad \&\right. \\
& \left.\bar{X}_{w}\left[0, \frac{1}{2}\right] \leqq b^{\prime}\right)
\end{aligned}
$$

(say $I J$ ). In terms of conditional probability

$$
I=P\left(\underline{Y}_{w}^{\prime}\left[0, \frac{1}{2}\right] \geqq-\Delta\right) P\left(\underline{X}_{w}^{\prime}\left[0, \frac{1}{2}\right] \geqq a \quad \& \quad Z_{w}\left(\frac{1}{2}\right) \in D \mid \underline{Y}_{w}^{\prime}\left[0, \frac{1}{2}\right] \geqq-\Delta\right)
$$

(say $\left.I_{1} I_{2}\right)$. Making use of the fact that $\tilde{Z}_{w}(t)=Z_{w}(1 / 2-t)-Z_{w}(1 / 2),-\infty<t<\infty$, is also a standard Brownian motion, we have

$$
\begin{aligned}
& J=P\left(\underline{Y}_{w}\left[0, \frac{1}{2}\right] \geqq-\delta \&-c \leqq Y_{w}\left(\frac{1}{2}\right) \leqq \frac{1}{2}-c \&\right. \\
& \left.\bar{X}_{w}\left[0, \frac{1}{2}\right] \leqq b^{\prime}+X_{w}\left(\frac{1}{2}\right)\right)=P\left(\underline{Y}_{w}\left[0, \frac{1}{2}\right] \geqq-\delta\right) P\left(-c \leqq Y_{w}\left(\frac{1}{2}\right) \leqq \frac{1}{2}-c \&\right. \\
& \left.\bar{X}_{w}\left[0, \frac{1}{2}\right] \leqq b^{\prime}+X_{w}\left(\frac{1}{2}\right) \mid \underline{Y}_{w}\left[0, \frac{1}{2}\right] \geqq-\delta\right)
\end{aligned}
$$

(say $J_{1} J_{2}$ ). Then, by (3.1), (3.2) and by (3.3) we get $I_{1} \asymp \Delta(\Delta \rightarrow+0)$ and $J_{1} \asymp \delta$ $(\delta \rightarrow+0)$. Moreover, it follows from the limit theorem of conditioned Brownian motion (see, Shimura [3]) both $I_{2}$ and $I_{3}$ tend to positive numbers as $\Delta \rightarrow+0$ and $\delta \rightarrow+0$ respectively. Hence we have (3.5) from (3.6).

We can show (3.5) for the case $\pi / 2<\theta \leqq \pi$ in a similar way, so we omit it here. 


\section{$\S 4$. Proof of Theorem 1 .}

In this section $K_{1}, K_{2}, \cdots$ will denote some positive constants. Note that $P\left(A_{k}^{n}\right)=P\left(A_{1}^{n}\right)$ for $1 \leqq k \leqq 2 n$, because the Brownian motion has stationary increments. Then we have from Lemma 1

$$
E\left(S_{2 n}^{n}\right)=\sum_{k=1}^{2 n} P\left(A_{k}^{n}\right) \leqq 2 n\left(K_{1} / n\right)=2 K_{1}<\infty .
$$

Set $B_{k}^{n}=A_{k}^{n}-\bigcup_{j=1}^{k-1} A_{j}^{n}$, and denote by $C_{k}^{n}$ the event

$$
\begin{aligned}
\bigcap_{j=1}^{k-1}\left\{w \in W \mid \underline{Y}_{w}\left[\frac{j}{n}-2, \frac{j-1}{n}\right]<\underline{Y}_{w}\left[\frac{j-1}{n}, \frac{j}{n}\right]\right. \text { or } \\
\left.\qquad \underline{Y}_{w}^{\prime}\left[\frac{j-1}{n}, \frac{j}{n}\right]>\underline{Y}_{w}^{\prime}\left[\frac{j}{n}, \frac{k}{n}\right]\right\} .
\end{aligned}
$$

Let $F_{k}^{n}(x)$ denote the conditional probability distribution function

$$
P\left(\underline{Y}_{w}\left[\frac{k}{n}-2, \frac{k-1}{n}\right] \geqq \underline{Y}_{w}\left[\frac{k-1}{n}, \frac{k}{n}\right] \& Y_{w}^{\prime}\left(\frac{k}{n}\right)-\underline{Y}_{w}^{\prime}\left[\frac{k-1}{n}, \frac{k}{n}\right] \leqq x \mid C_{k}^{n}\right) .
$$

Note that $B_{k}^{n}=C_{k}^{n} \cap A_{k}^{n}$ and that $C_{k}^{n}$ is an event given in terms of $Z_{w}(s)$, $-\infty<s \leqq k / n$. Then, making use of the independence and the stationarity of the increments, we have

$$
P\left(B_{k}^{n}\right)=P\left(C_{k}^{n}\right)\left\{\int_{0}^{(j-1 / n)^{1 / 2}}+\int_{(j-1 / n)^{1 / 2}}^{\infty}\right\} d F_{k}^{n}(x) P\left(\underline{Y}_{w}^{\prime}\left[0,2-\frac{1}{n}\right] \geqq-x\right) .
$$

Then, as was shown in [1] (7.23), we conclude from (3.1), (3.2) and from (3.4) the following:

$$
P\left(B_{k}^{n}\right)<2 P\left(C_{k}^{n}\right) \int_{0}^{(j-1 / n)^{1 / 2}} x d F_{k}^{n}(x)
$$

for every $k$ and $j$ satisfying

$$
1 \leqq k \leqq n \text { with } P\left(B_{k}^{n}\right) \geqq 2 / n^{2} \text { and } 6 \log n+1 \leqq j \leqq 2 n-k
$$

(note $P\left(B_{1}^{n}\right)=P\left(A_{1}^{n}\right) \geqq 2 / n^{2}$ for almost all $n$ by Lemma 1 ).

Noting $B_{k}^{n} \cap A_{k+j}^{n}=C_{k}^{n} \cap A_{k}^{n} \cap A_{k+j}^{n}$, we have

$$
\begin{aligned}
& P\left(B_{k}^{n} \cap A_{k+j}^{n}\right) \geqq P\left(C_{k}^{n} \& \underline{Y}_{w}\left[\frac{k}{n}-2, \frac{k-1}{n}\right] \geqq \underline{Y}_{w}\left[\frac{k-1}{n}, \frac{k}{n}\right] \&\right. \\
& \underline{Y}_{w}^{\prime}\left[\frac{k-1}{n}, \frac{k}{n}\right] \leqq \underline{Y}_{w}^{\prime}\left[\frac{k}{n}, \frac{k+j}{n}\right] \& \\
& \underline{Y}_{w}^{\prime}\left[\frac{k+j-1}{n}, \frac{k+j}{n}\right] \geqq Y_{w}^{\prime}\left(\frac{k+j-1}{n}\right)-\left(\frac{j-1}{n}\right)^{1 / 2} \geqq Y_{w}^{\prime}\left(\frac{k}{n}\right) \& \\
& \underline{Y}_{w}\left[\frac{k-1}{n}, \frac{k+j-1}{n}\right] \geqq \underline{Y}_{w}\left[\frac{k+j-1}{n}, \frac{k+j}{n}\right] \&
\end{aligned}
$$




$$
\begin{aligned}
& \underline{Y}_{w}\left[\frac{k-1}{n}, \frac{k}{n}\right] \geqq Y_{w}\left(\frac{k}{n}\right)-\left(\frac{j-1}{n}\right)^{1 / 2} \geqq Y_{w}\left(\frac{k+j-1}{n}\right) \& \\
& \left.\underline{Y}_{w}^{\prime}\left[\frac{k+j-1}{n}, \frac{k+j}{n}\right] \leqq \underline{Y}_{w}^{\prime}\left[\frac{k+j}{n}, \frac{k+j-1}{n}+2\right]\right) \\
& >P\left(C_{k}^{n} \& \underline{Y}_{w}\left[\frac{k}{n}-2, \frac{k-1}{n}\right] \geqq \underline{Y}_{w}\left[\frac{k-1}{n}, \frac{k}{n}\right] \&\right. \\
& \underline{Y}_{w}^{\prime}\left[\frac{k-1}{n}, \frac{k}{n}\right] \leqq \underline{Y}_{w}^{\prime}\left[\frac{k}{n}, \frac{k+j-1}{n}\right] \& Y_{w}^{\prime}\left(\frac{k+j-1}{n}\right)-Y_{w}^{\prime}\left(\frac{k}{n}\right) \geqq\left(\frac{j-1}{n}\right)^{1 / 2} \& \\
& \underline{Y}_{w}\left[\frac{k}{n}, \frac{k+j-1}{n}\right] \geqq \underline{Y}_{w}\left[\frac{k+j-1}{n}, \frac{k+j}{n}\right] \& Y_{w}\left(\frac{k+j-1}{n}\right)-Y_{w}\left(\frac{k}{n}\right) \\
& \left.\leqq-\left(\frac{j-1}{n}\right)^{1 / 2} \& \underline{Y}_{w}^{\prime}\left[\frac{k+j-1}{n}, \frac{k+j}{n}\right] \leqq \underline{Y}_{w}^{\prime}\left[\frac{k+j}{n}, \frac{k+j-1}{n}+2\right]\right) \\
& -P\left(\underline{Y}_{w}\left[\frac{k-1}{n}, \frac{k}{n}\right]<Y_{w}\left(\frac{k}{n}\right)-\left(\frac{j-1}{n}\right)^{1 / 2}\right)-P\left(\underline{Y}_{w}^{\prime}\left[\frac{k+j-1}{n}, \frac{k+j}{n}\right]\right. \\
& \left.<Y_{w}^{\prime}\left(\frac{k+j-1}{n}\right)-\left(\frac{j-1}{n}\right)^{1 / 2}\right)
\end{aligned}
$$

(say $L_{1}-L_{2}-L_{3}$ ). We apply the independence and the stationarity of the increments repeatedly to get the following estimate of $L_{1}$ :

$$
\begin{aligned}
& L_{1}>P\left(C_{k}^{n}\right) \int_{0}^{\infty} d F_{k}^{n}(x) P\left(\underline{Y}_{w}^{\prime}\left[0, \frac{j-1}{n}\right] \geqq-x \& Y_{w}^{\prime}\left(\frac{j-1}{n}\right) \geqq\left(\frac{j-1}{n}\right)^{1 / 2} \&\right. \\
& \underline{Y}_{w}\left[0, \frac{j-1}{n}\right]-Y_{w}\left(\frac{j-1}{n}\right) \geqq-n^{-1 / 2} \& \underline{Y}_{w}\left[\frac{j-1}{n}, \frac{j}{n}\right]-Y_{w}\left(\frac{j-1}{n}\right) \leqq-n^{-1 / 2} \\
& \left.\& Y_{w}\left(\frac{j-1}{n}\right) \leqq-\left(\frac{j-1}{n}\right)^{1 / 2} \& \underline{Y}_{w}^{\prime}\left[\frac{j-1}{n}, \frac{j}{n}\right] \leqq \underline{Y}_{w}^{\prime}\left[\frac{j}{n}, \frac{j-1}{n}+2\right]\right) \\
& >P\left(C_{k}^{n}\right) \int_{0}^{(j / n-1 / n)^{1 / 2}} d F_{k}^{n}(x) G\left(\left(\frac{n}{j-1}\right)^{1 / 2} x,(j-1)^{-1 / 2}\right) P\left(\underline{Y}_{w}[0,1] \leqq-1 \&\right. \\
& \left.\underline{Y}_{w}^{\prime}[0,1] \leqq-1 \& Y_{w}^{\prime}(1) \geqq 1\right) P\left(\underline{Y}_{w}^{\prime}\left[0,2-\frac{1}{n}\right] \geqq-2 n^{-1 / 2}\right) .
\end{aligned}
$$

By (3.1), (3.2) and by (3.3)

$$
P\left(\underline{Y}_{w}^{\prime}\left[0,2-\frac{1}{n}\right] \geqq-2 n^{-1 / 2}\right) \asymp n^{-1 / 2}(n \rightarrow \infty),
$$

and by (3.4)

$$
L_{2}=L_{3}=P\left(\underline{Y}_{w}[0,1]<-(j-1)^{1 / 2}\right) \leqq n^{-3} \text { for } j \geqq 6 \log n+1 .
$$

Then it follows from (4.4), (4.5) and from Lemma 2 the following: 


$$
P\left(B_{k}^{n} \cap A_{k+j}^{n}\right)>K_{2} \frac{P\left(C_{k}^{n}\right)}{j-1} \int_{0}^{(j / n-1 / n)^{1 / 2}} x d F_{k}^{n}(x)-2 n^{-3} \text { for } j \geqq 6 \log n+1 .
$$

Therefore, from (4.2) and (4.6) we have

$$
P\left(A_{k+j}^{n} \mid B_{k}^{n}\right)>K_{3}(j-1)^{-1}-n^{-1}
$$

for every $k$ and $j$ satisfying (4.3) (see (7.24) in [1]).

Once we have (4.7), we can show in the same way to [1] p. 115 the following :

$$
E\left(S_{2 n}^{n} \mid S_{n}^{n} \geqq 1\right)>K_{4} \log n \text { for all } n,
$$

from which, together with (4.1), we conclude (2.1). This proves the theorem.

Concluding Remark. Suppose that $\boldsymbol{u}_{1}=\boldsymbol{u}_{2}(=\boldsymbol{u})$. Then $G(\Delta, \delta)=0$ for every $\Delta$ and $\delta$, because the processes $Y$ and $Y^{\prime}$ are coincident. So, in the case, we note that the right hand side of (4.5) vanishes and that, as a result, we do not have $\lim _{n \rightarrow \infty} E\left(S_{2 n}^{n} \mid S_{n}^{n} \geqq 1\right)=\infty$ which would lead an contradictory assertion $\mathscr{M}_{w}(\boldsymbol{u}, \boldsymbol{u})=\varnothing$ a. s..

\section{REFERENCES}

[1] A. Dvoretzky, P. Erdös And S. Kakutani, Nonincrease everywhere of the Brownian motion process, Proc. Fourth Berkeley Sympos. Math. Statist. and Probab. Vol. II (1961), 103-116.

[2] S. N. Evans, On the Hausdorff dimension of Brownian cone points, Math. Proc. Camb. Phil. Soc. 98 (1985), 343-353.

[3] M. Shimura, Excursions in a cone for two-dimensional Brownian motion, J. Math. Kyoto Univ. 25 (1985), 433-443.

College of Liberal Arts

TOHO UnIVERSITY

Miyama 2-2-1, Funabashi

Chiba 274 JAPAN 